\title{
Supersymmetric dark matter with gaugino non-universality
}

\author{
Nicolao Fornengo*† \\ Department of Theoretical Physics, University of Torino and INFN - Torino \\ via P. Giuria 1, I-10125 Torino \\ E-mail: ifornengo@to.infn.it' \\ Web: 'WWW.to.infn.it/ fornengo, WWW.astroparticle.to.infn.it
}

ABSTRACT: In the frame of an effective MSSM model without gaugino-mass unification at a grand unification scale, we discuss the phenomenology of relic neutralinos which arise to be lighter than in the in the Minimal Supersymmetric Standard Model. The calculation of the relic abundance of these light neutralinos, when confronted with the recent WMAP results on the dark matter content of the Universe, allows us to set an absolute lower bound on the neutralino mass: $m_{\chi} \gtrsim 6 \mathrm{GeV}$ for a light pseudoscalar higgs, and $m_{\chi} \gtrsim 22 \mathrm{GeV}$ for $m_{A} \gtrsim 180 \mathrm{GeV}$. We then discuss the prospects of detection of these light relic neutralinos: they can provide detectable rates in direct detection experiments and a detectable antiproton flux from annihilation in the galactic halo. The gamma-ray signal can reach detectable levels only for strong singular dark matter density profiles.

\section{Introduction}

Typical assumptions in supersymmetric models consider a unification condition for the gaugino masses $M_{1,2,3}$ at the GUT scale. This hypothesis implies that at the electroweak scale, $M_{1} \simeq 0.5 M_{2}$. Under this unification condition the usual bound on the neutralino mass $m_{\chi} \gtrsim 50 \mathrm{GeV}$ is derived from the lower bound on the chargino mass determined at LEP2 $\left(m_{\chi}^{ \pm} \gtrsim 100 \mathrm{GeV}\right)$. In the case where $M_{1} \equiv R M_{2}$, with $R<0.5$, the neutralino can be lighter than in the gaugino-universal models and accelerator experiments cannot set an absolute lower bound on $m_{\chi}$, since they fix the chargino mass, which depends on $M_{2}$ but not on $M_{1}$.

We consider here an extension of the MSSM allowing a deviation from gauginouniversality by the introduction of the parameter $R$, varied here in the interval: $(0.01 \div 0.5)$.

\footnotetext{
${ }^{*}$ Speaker.

${ }^{\dagger}$ In collaboration with A. Bottino, F. Donato and S. Scopel
} 
We therefore employ an effective MSSM scheme at the electroweak scale, defined in terms of a minimal number of parameters, only those necessary to shape the essentials of the theoretical structure of MSSM and of its particle content. The assumptions that we impose at the electroweak scale are: a) all squark soft-mass parameters are taken degenerate: $m_{\tilde{q}_{i}} \equiv m_{\tilde{q}} ;$ b) all slepton soft-mass parameters are taken degenerate: $m_{\tilde{l}_{i}} \equiv m_{\tilde{l}}$; c) all trilinear parameters are set to zero except those of the third family, which are defined in terms of a common dimensionless parameter $A: A_{\tilde{b}}=A_{\tilde{t}} \equiv A m_{\tilde{q}}$ and $A_{\tilde{\tau}} \equiv A m_{\tilde{l}}$. As a consequence, the supersymmetric parameter space consists of the following independent parameters: $M_{2}, \mu, \tan \beta, m_{A}, m_{\tilde{q}}, m_{\tilde{l}}, A$ and $R \equiv M_{1} / M_{2}$. In the previous list of parameters we have denoted by $\mu$ the Higgs mixing mass parameter, by $\tan \beta$ the ratio of the two Higgs v.e.v.'s and by $m_{A}$ the mass of the CP-odd neutral Higgs boson.

In our numerical analysis we have varied the MSSM parameters within the following ranges: $1 \leq \tan \beta \leq 50,100 \mathrm{GeV} \leq|\mu|, M_{2}, m_{\tilde{q}}, m_{\tilde{l}} \leq 1000 \mathrm{GeV}, \operatorname{sign}(\mu)=-1,1,90 \mathrm{GeV} \leq$ $m_{A} \leq 1000 \mathrm{GeV},-3 \leq A \leq 3$, for a sample of representative values of $R$ in the range $0.01 \leq R \leq 0.5$. This range for $R$, implemented with the experimental lower limit on $M_{2}$ of about $100 \mathrm{GeV}$, implies that the lower bound on the neutralino mass can be moved down to few GeV's for $R \sim 0.01$. The ensuing light neutralinos have a dominant bino component; a deviation from a pure bino composition is mainly due to a mixture of $\tilde{B}$ with $\tilde{H}_{1}^{\circ}$. This is shown in Fig. as the non-universality parameter $R$ increases.

New data on the cosmic microwave background [i] other cosmological observations, are progressively narrowing down the ranges of the relic abundances for dark matter and the range of $\Omega_{C D M} h^{2}$ is reaching a unprecedented level of accuracy. From Ref. 流, we obtain a $2 \sigma$ range: $0.095 \leq \Omega_{C D M} h^{2} \leq 0.131$. The relic abundance $\Omega_{\chi} h^{2}$ of neutralinos lighter than $50 \mathrm{GeV}$ which arise in our class of gaugino non-universal models has a relatively simple structure in terms of dominant diagrams in

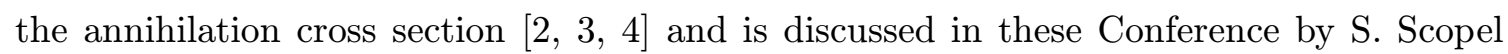
[5]. Here I just remind the remarkable fact that combining our calculation of the relic abundance of light neutralinos with the recent determination on $\Omega_{C D M} h^{2}$, we have been able to set an absolute lower bound on the neutralino mass: when $A$ is light a lower bound

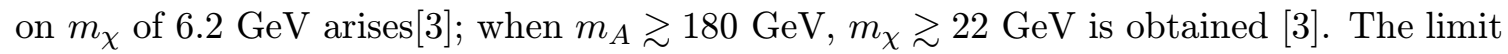
obtained for a light $A$ bosons is mildly dependent on the quark-hadron phase transition temperature $T_{Q C D}$ in the early Universe, since in this case very light neutralinos may decouple after or before $T_{Q C D}$, depending whether the decoupling temperature is lower or higher than $T_{Q C D}$ [3in]. It is remarkable that a lower limit on $m_{\chi}$ is set not by searches at accelerators, but instead by cosmological arguments.

\section{Flux of antiprotons}

Relic neutralinos bounded to form the halo of our Galaxy may annihilate and produce potential signals. One possibility is the production of an antiproton flux [i] $\left.{ }_{1}^{1} \overline{1}\right]$. The details of the calculation of the primary antiproton flux is found in Ref. [i $[\bar{i}]$, and details on the calculation of the secondary antiproton component in cosmic rays is discussed in Refs. 

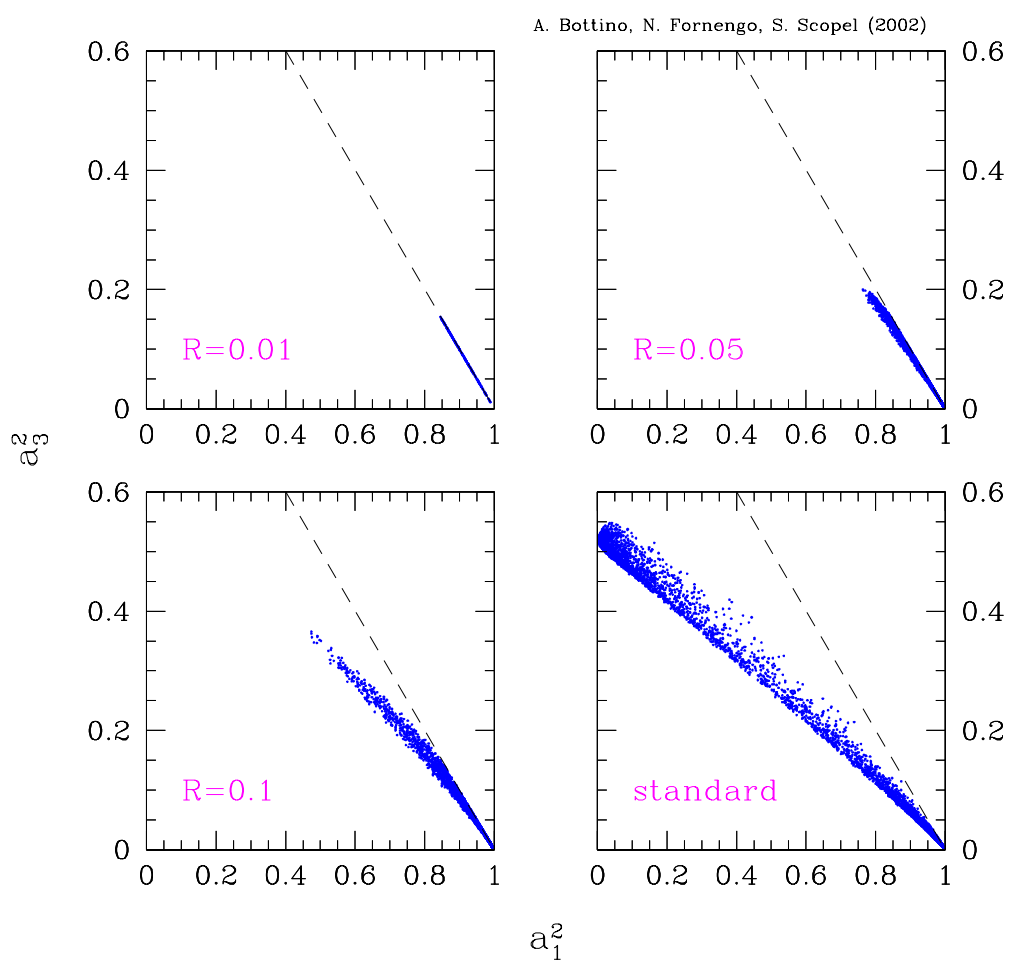

Figure 1: Scatter plots of the neutralino composition in terms of $\tilde{B}\left(a_{1}\right)$ and of $\tilde{H}_{1}^{\circ}\left(a_{3}\right)$ for $R=0.01,0.05,0.1$ and for the standard value $R=5 / 3 \tan ^{2} \theta_{W} \simeq 0.5$. The dashed lines denote the line where $a_{1}^{2}+a_{3}^{2}=1$.

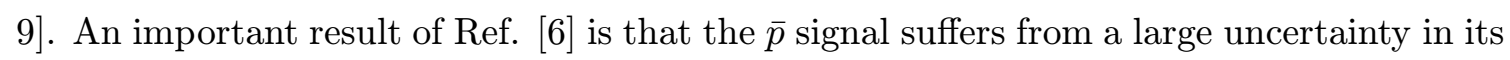
determination, due to aspects related to the propagation of cosmic rays in the Galaxy. Fig. $i_{2}^{2}$ shows an example of this uncertainty, related to one of the most important astrophysical parameter for antiproton diffusion in the halo, namely the height $L$ of the diffusive region. We see that the antiproton flux may suffer a two orders of magnitude uncertainty when $L$ is

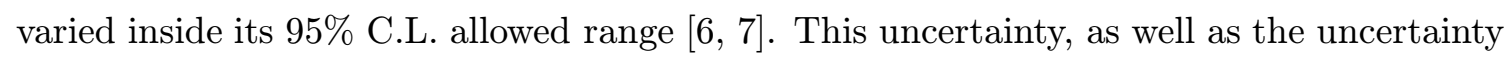
coming from the other relevant parameters like the diffusion coefficient, the galactic wind velocity, the Alfven velocity which takes into account reacceleration, and the spallation cross sections which determine the destruction of antiprotons through collisions with the interstellar medium, reflects in a large uncertainty in the predicted fluxes. This can be seen in Fig. $\overline{3}$. The same figure shows that a much smaller uncertainty affects the standard secondary cosmic rays. They are produced mainly in the disk of the Galaxy, where also

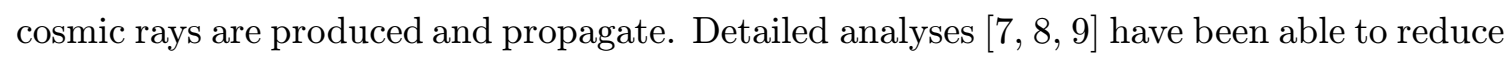
the uncertainties on the disk components, by showing that, although the cosmic ray data are not able to fix any of the diffusion and propagation parameters alone, they nevertheless 

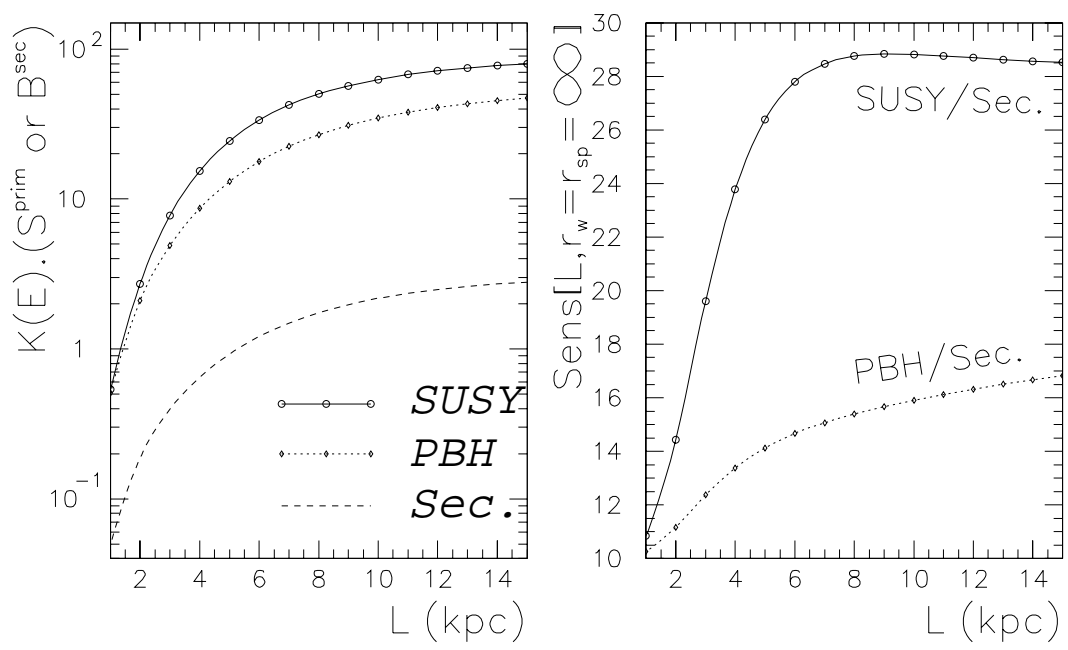

Figure 2: This plot displays the quantities $K(E) \times S_{\text {astro }}^{\text {prim }}$ (left panel), which is proportional to the antiproton flux (at high energies) and $\mathcal{S}$ ens (right panel), which is the ratio between the primary and secondary antiproton fluxes, as a function of the propagation parameter $L\left(r_{\mathrm{w}}=r_{\mathrm{sp}}=\infty\right.$, i.e. no wind, no spallations) for an isothermal profile. For and exact definition of the two quantities, see

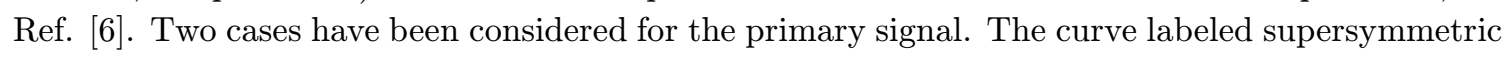
corresponds to an effective source term proportional to the square of the dark matter density profile, whereas the curve PBH corresponds to a source term linearly proportional to the dark matter density profile, as for primordial black holes.

force them to align along specific trajectories in their parameter space. Secondaries depend on the astrophysical parameters along the same trajectories and therefore they are well determined. On the contrary the primary flux, which is mostly produced in the galactic halo and propagate more deeply into it, breaks those degeneracies and suffers of larger uncertainties. We are therefore facing an almost two order of magnitude uncertainty on the primary antiproton flux: this limits somehow our capability of using antiprotons as a tool for searching for dark matter. We remind that antideuterons instead offer a much better possibility, since they allow for a much more clear signature at low kinetic energies [1] $1 \overline{1} \overline{0} \overline{1}]$.

Fig. 'Ais shows the comparison of some of our predicted fluxes with the current data on antiprotons in cosmic rays. The primary fluxes are calculated for the choice of astrophysical parameters which gives the median curve in Fig. supersymmetric parameter space with the data in Fig. $\overline{1}$. The figure shows the antiproton flux calculated in the lowest energy bin where data are available, for the median choice of astrophysical parameters. We see that all the predicted fluxes for neutralino masses below $20 \mathrm{GeV}$ could be excluded, since they provide fluxes in excess of data. However, we have to remind the large uncertainty in the primary flux calculation which comes from the astrophysical parameters related to diffusion and propagation: the use of the most 


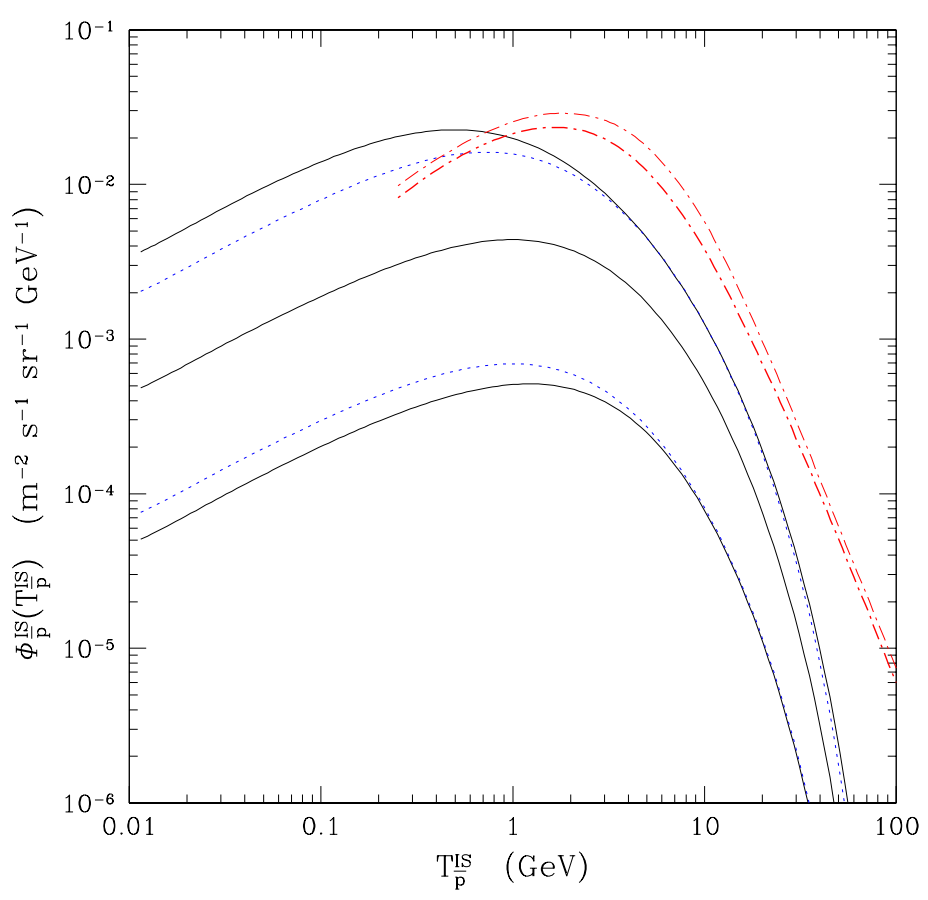

Figure 3: The solid lines represent the antiproton flux for a $m_{\chi}=100 \mathrm{GeV}$ neutralino and for maximal, median and minimal astrophysical configurations, for $\chi_{\mathrm{B} / \mathrm{C}}^{2} \leq 40$. Dotted lines: the same, but for for $\chi_{\mathrm{B} / \mathrm{C}}^{2} \leq 30$. The dot-dashed band corresponds to the secondary flux as taken from Ref. [i]] for all the configurations giving $\chi_{B / C}^{2} \leq 40$.

conservative set of astrophysical parameters gives the results shown in Fig. ' ${ }^{6} \overline{-}_{i}^{-}$It is not possible, at the moment, to exclude supersymmetric configurations. Neutralinos of masses around $10 \mathrm{GeV}$ are nevertheless close to a possible detection or seclusion in the antiproton channel.

\section{Flux of gamma rays from the galactic center}

Another interesting signal is the production of gamma-rays. The largest signal is expected to come from the galactic center, where the dark matter density is likely to be the largest. This signal is therefore very dependent on the shape of the inner parts of the galactic halo. Fig. $\bar{i}_{i}$ shown the line-of-sight integral of the dark matter density profile from our position in the galaxy toward the center, for three different shapes of the halo: very steep profiles, like the one predicted by the numerical simulations of Moore et al. can produce a large 


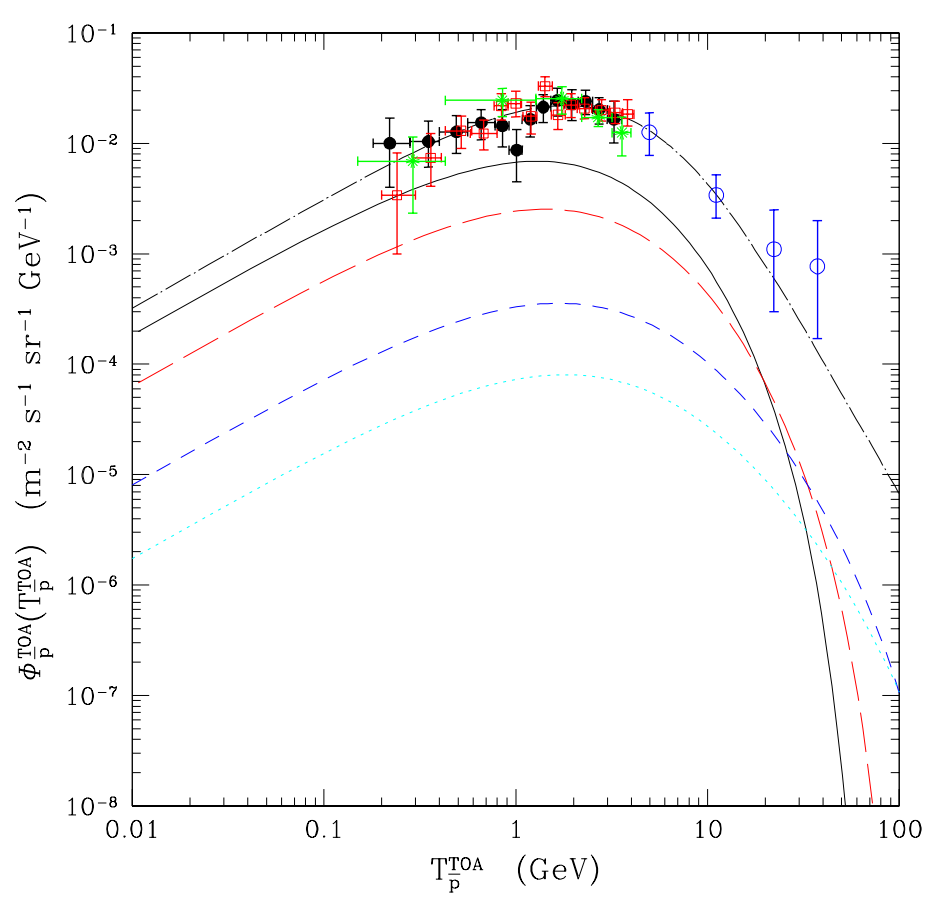

Figure 4: Primary TOA antiproton fluxes as a function of the antiproton kinetic energy, for representative spectra from neutralino annihilation. The solid line refers to $m_{\chi}=60 \mathrm{GeV}$, the long-dashed line to $m_{\chi}=100 \mathrm{GeV}$, the short-dashed line to $m_{\chi}=300 \mathrm{GeV}$ and the dotted line to $m_{\chi}=500 \mathrm{GeV}$. The astrophysical parameters correspond to the median choice. Solar modulation is calculated for a period of minimal solar activity. The upper dot-dashed curve corresponds to the

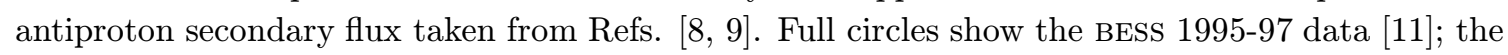

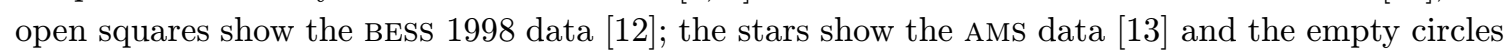
show the CAPRICE data [1] 14 in].

flux of gamma rays. Nowadays the Moore et al. behavior is somehow questioned, but a singular slope, even steep, is nevertheless not excluded at present.

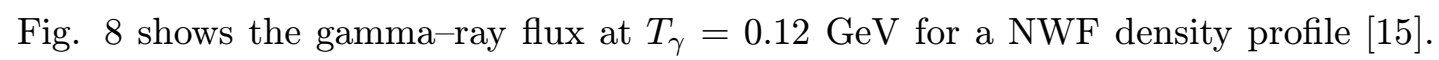
The scatter plot is compared to the EGRET [i] $\overline{1} \overline{0}]$ measurement in that energy bin, which is supposed to be compatible with background estimates [i] $\left.{ }_{1}^{1} \overline{\sigma_{i}}\right]$. No excess is therefore required in that bin. The calculations show that the expected signal fluxes are not incompatible with the data, and therefore no supersymmetric configuration is excluded. A steeper density profile, like the Moore et al. could go in conflict with observations only for very low neutralino masses. On the other hand, at larger photon energies, EGRET observed an

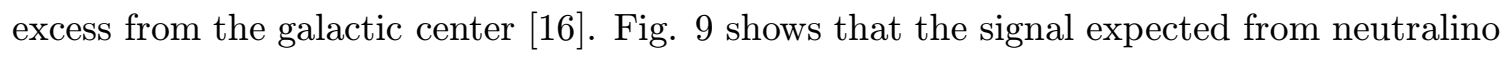




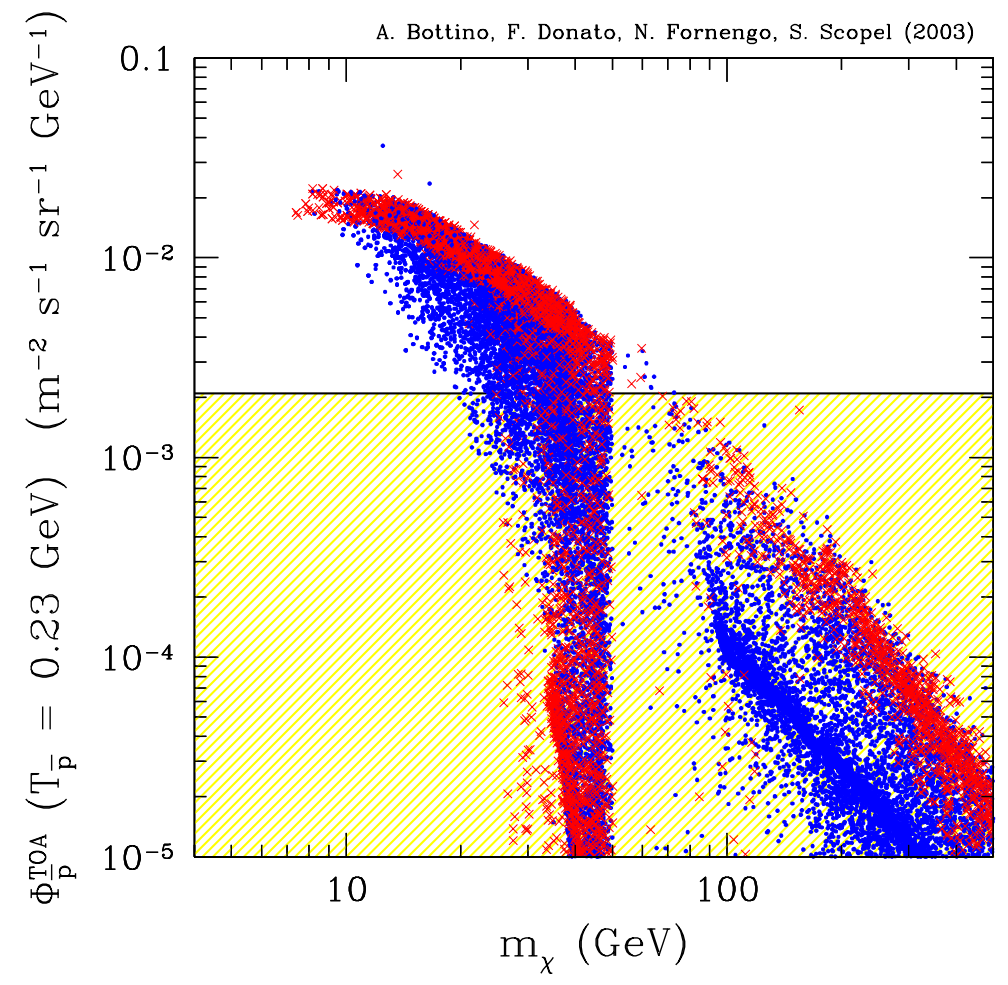

Figure 5: Antiproton flux at $T_{\bar{p}}=0.23 \mathrm{GeV}$ as a function of the neutralino mass, for a full scan of the gaugino non-universal supersymmetric model. Crosses (red) and dots (blue) denote neutralino configurations with $0.095 \leq \Omega_{\chi} h^{2} \leq 0.131$ and $\Omega_{\chi} h^{2}<0.095$. The shaded region denotes the amount of primary antiprotons which can be accomodated at $T_{\bar{p}}=0.23 \mathrm{GeV}$ without entering in conflict with experimental data and secondary antiproton calculations. The best fit for the astrophysical parameters is used.

annihilation is not able to explain the excess, for a NFW distribution. A steeper density profile, like the one predicted by Moore et al., would instead allow the low-mass neutralino around 6-10 GeV to reach the level expected from the EGRET excess 這的. Notice that is possible without going in conflict with the lower energy bin shown in Fig.

In conclusion, for the gamma-ray signal we need a sizable dark matter overdensity in order to explain the excess observed by EGRET. This overdensity may be due either to a very steep density profile at the galactic center or to a clumpy mass distribution toward the galactic center.

\section{Conclusions}

We have discussed the phenomenology of light relic neutralinos which arise in a class of supersymmetric models where gaugino-universality is violated. Since accelerator searches 


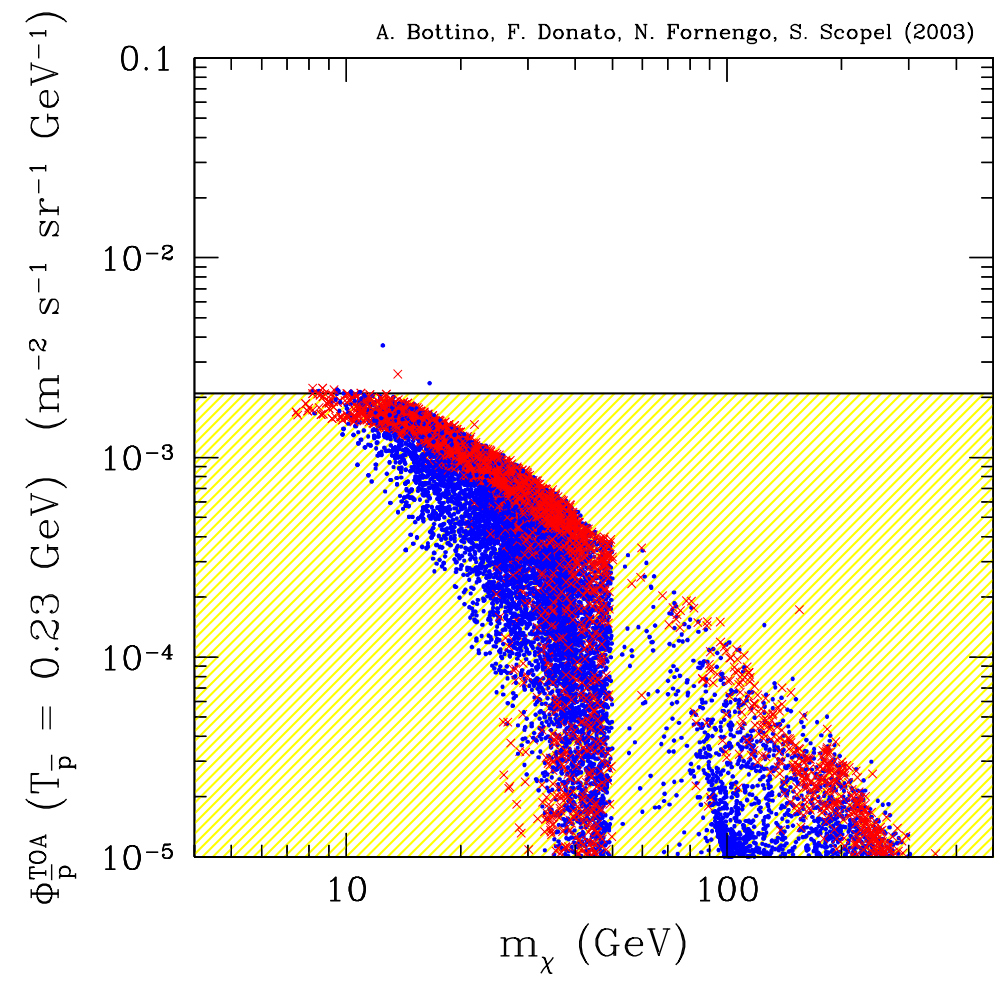

Figure 6: The same as in Fig. ${ }_{2}^{1}$, except for the choice of astrophysical parameters, which now provide the more conservative antiproton fluxes.

of supersymmetric particles are mainly constraining the chargino mass, it turns out that the neutralino mass in this class of models is practically lacking of a lower bound. The fact that they are mostly binos, makes difficult the possibility to constrain them through the invisible $Z$ decay amplitude. The conclusion is that accelerator physics is not currently able to exclude the possibility of neutralinos in the $\mathrm{GeV}$ mass range.

On the other hand, the recent precise determination of the amount of dark matter in the Universe, coming from WMAP, galaxy surveys and Lyman- $\alpha$ studies, constrains the value of $\Omega_{C D M} h^{2}$ to be smaller than 0.131. From this limit, we have been able to set an absolute lower bound on the mass of the light neutralinos: the limit is $6.2 \mathrm{GeV}$ for light pseudoscalar higgses, and $22 \mathrm{GeV}$ for $m_{A} \gtrsim 180 \mathrm{GeV}$. This limit relies only on cosmological analysis. The discussion of this relevant point, as well as the analysis of the prospects of detection of these light neutralinos by means of direct detection, are discussed in Scopel's

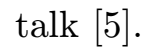

As for the indirect signals due to neutralino annihilation in the halo, we have here discussed the possibility to detect a diffuse flux of antiprotons ad a gamma-ray flux coming from the galactic center. We have shown that the antiproton flux, although not very 


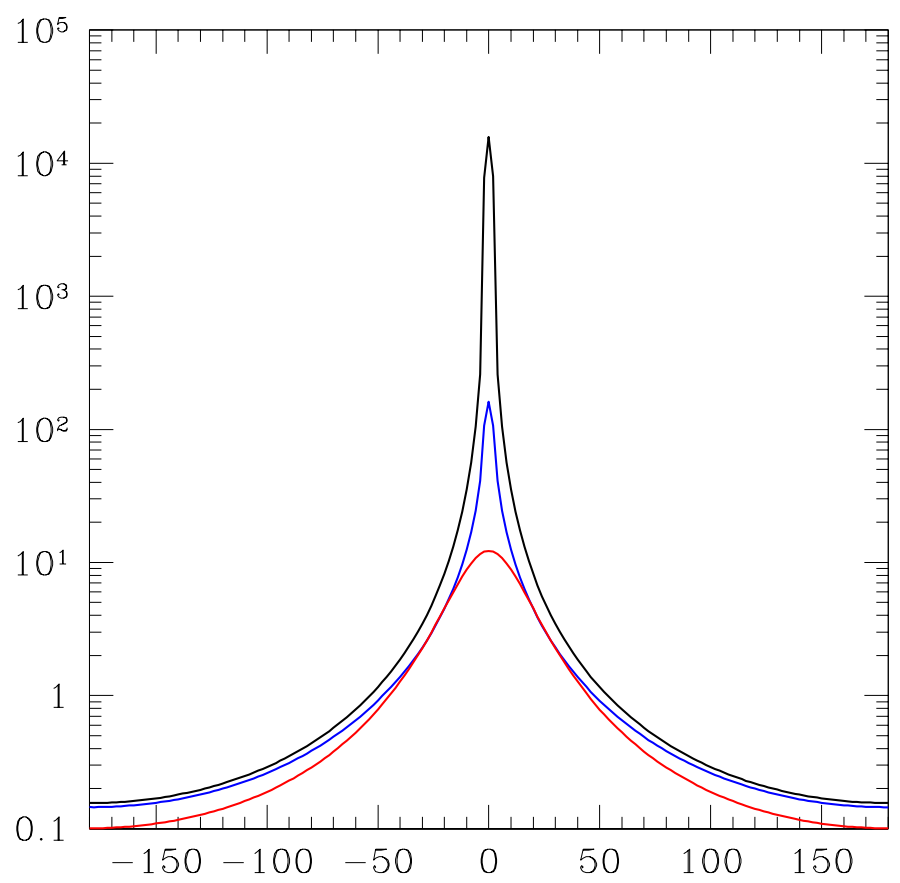

Figure 7: Line-of-sight integral of the squared of the dark matter density profile toward the galactic center, along the galactic plane. The gamma-ray flux is proportional to this quantity. The abscissa shows the galactic longitude as seen from the Earth position. The lowest curve corresponds to a cored isothermal profile, the median curve to a NFW density profile $\left[\rho(r) \longrightarrow r^{-1}\right.$ as $\left.r \rightarrow 0\right]$, the highest curve to a Moore et al. profile $\left[\rho(r) \longrightarrow r^{-1.5}\right.$ as $\left.r \rightarrow 0\right]$. A cut off $r_{c}=0.01 \mathrm{pc}$ has been used in the inner parts of the halo (below $r_{c}$ the density is constant).

sensitive to the dark matter density profile, is instead very dependent on the uncertainties on the diffusion and propagation parameters which describe the diffusive halo of the Galaxy. This limits the capabilities for this kind of searches, since the uncertainty on the primary flux is close to a factor of 100 at low antiproton kinetic energies (while the primary flux is theoretically known at the level of $25 \%$ or better). However, it is tempting the fact that the predicted rates are at the level of the experimental sensitivities and may already allow detection of (or to set constraints on) supersymmetric models.

The flux of gamma rays coming from the galactic center is very sensitive to the inner properties of the dark matter halo. Depending on the actual density profile, the fluxes can change by up to 2.5 orders of magnitude. The gamma ray flux is potentially relevant for dark matter detection for very light neutralinos, in the $6-25 \mathrm{GeV}$ mass range, but only for very steep density profile, or for a clumpy mass distribution where a sizable distribution of 


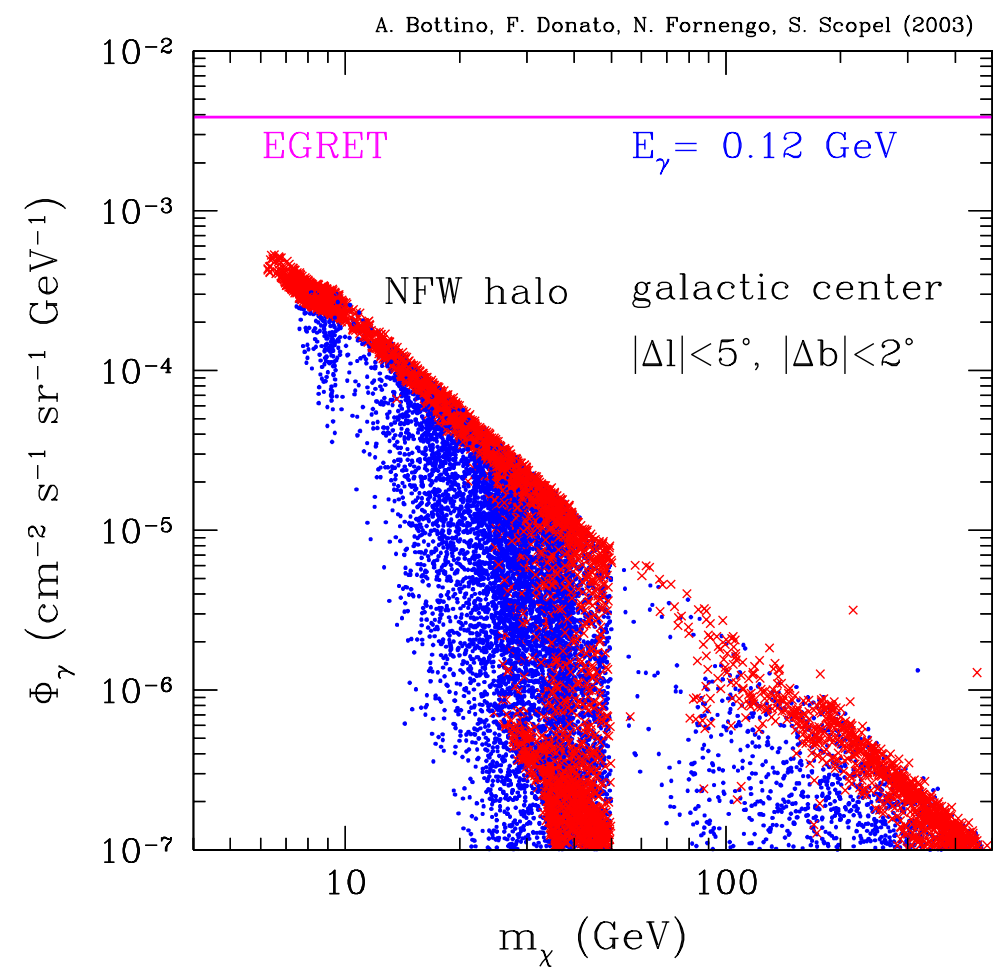

Figure 8: Gamma ray flux from the galactic center for a NFW matter density profile, for $E_{\gamma}=0.12$ $\mathrm{GeV}$. Crosses (red) and dots (blue) denote neutralino configurations with $0.095 \leq \Omega_{\chi} h^{2} \leq 0.131$ and $\Omega_{\chi} h^{2}<0.095$.

clumps occurs along the line of sight directed toward the galactic center. For the gamma ray flux, moreover, sources of uncertainties on both the signal and the background have to better understood.

\section{References}

[1] D.N. Spergel et al., Ap. J. Suppl. 148 (2003) 175.

[2] A. Bottino, N. Fornengo and S. Scopel, Phys. Rev. D 67 (2003) 063519.

[3] A. Bottino, F. Donato, N. Fornengo and S. Scopel, Phys.Rev. D 68 (2003) 043506.

[4] A. Bottino, F. Donato, N. Fornengo, S. Scopel, to appear in Phys. Rev. D [hep-ph/0307303].

[5] S. Scopel, these Proceedings.

[6] F. Donato, N. Fornengo, D. Maurin, P. Salati and R. Taillet, to appear in Phys. Rev. D [astro-ph/0306207].

[7] D. Maurin, F. Donato, R. Taillet, and P. Salati, Astrophys. J. 555 (2001 585. 


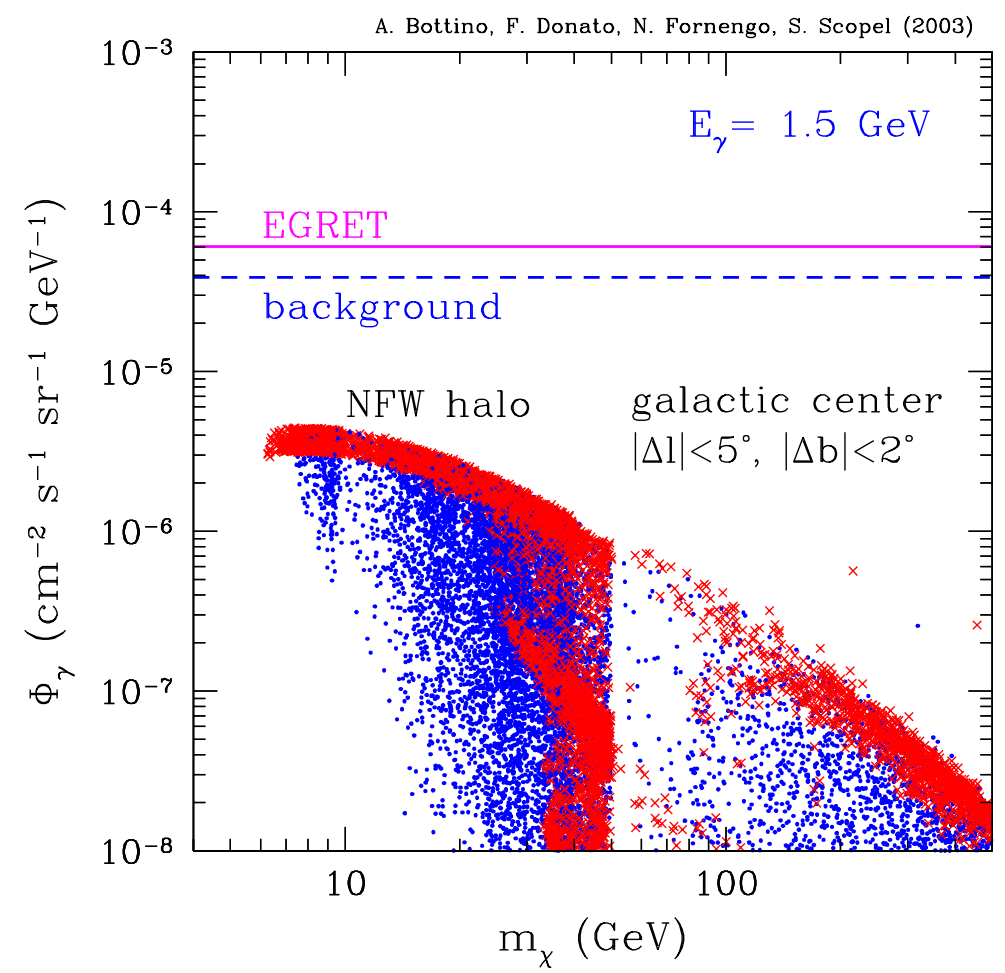

Figure 9: The same as in Fig. $\bar{p}_{\mathfrak{p}}^{\prime}$ for $E_{\gamma}=1.5 \mathrm{GeV}$.

[8] F . Donato, D. Maurin, P. Salati, A. Barrau, G. Boudoul, and R. Taillet, Astrophys. J. 563 (2001) 172.

[9] D. Maurin, R. Taillet, F. Donato, P. Salati, A. Barrau, and G. Boudoul, in research Signposts, Recent Developments in Astrophysics, astro-ph/0212111.

[10] F. Donato, N. Fornengo, P. Salati, Phys. Rev. D 62 (2000) 043003.

[11] S. Orito et al., (BESS Collaboration), Phys. Rev. Lett. 84 (2000) 1078.

[12] T. Maeno, et al. (BESS Collaboration), Astropart. Phys. 16 (2001) 121.

[13] M Aguilar, et al. (AMS Collaboration), Phys. Rep. 366 (2002) 331.

[14] M. Boezio, et al. (CAPRICE Collaboration), Astrophys. J. 561 (2001) 787.

[15] A. Bottino, F. Donato, N. Fornengo, S. Scopel, in preparation.

[16] S.D. Hunter et al., Ap. J. 481 (1997) 205. 